\title{
Voltage Distribution in Power Transformer Winding under Transient Impulse
}

\author{
Haifeng Ye ${ }^{1, a}$, Ya Gao ${ }^{1, b}$, Hao Wu ${ }^{1, c}$, Yabo Li ${ }^{2, d}$, Zhen Wu, ${ }^{2, e}$, Guoming Ma ${ }^{2, f}$ \\ ${ }^{1}$ Electric Power Research Institute of Guangdong Power Grid Co., Ltd , Guangzhou, China \\ ${ }^{2}$ North China Electric Power University, Beijing, China \\ a Yhf2007@126.com, bgaoya@gddky.csg.cn, c shouwangzhe789@163.com, dncepulun@126.com, \\ ewuzhen0907@foxmail.com, ${ }^{\mathrm{f}}$ ncepumgm@gmail.com
}

Keywords: power transformer, simulation model, voltages distribution, transient impulse Abstract. Field experience verifies that even when good insulation coordination studies and well-accepted insulation design practices are applied, power transformers suffer dielectric failure as reported in the literature. The analysis of the failures and prevention of transformer require comprehensive knowledge of the transient voltages distribution in transformer winding. So this paper constructs a transformer circuit model to calculate transient voltage distribution along the transformer winding, which is based on a multiconductor transmission line (MTL) model. The simulated transient voltage distribution of axial series capacitance and shunt capacitance to ground is analyzed and proved out in the paper, which are in accord with the existing conclusion.

\section{Introduction}

In service, the transformer insulation system is subjected continuously to operating voltages and occasionally to over-voltages [1-3]. Due to these transient voltages, transformers must be subjected to impulse tests to prove the Basic Insulation Level (a method of expressing the magnitude of the voltage surge that a transformer will tolerate without dielectric breakdown) according to specific standards. In view this point, it is significant to investigate transient over-voltages transient overvoltage by different methods [4-8]. G. B. Gharehpetain put forward a hybrid model of the black box model with the detailed model superimposed $\mathrm{m}$ segments, which solves the problem of using different structure types in the same coil [9]. Z. J. Wang proposed a multi-transmission line distribution parameter model and the double disk circle lumped parameter model of the hybrid model, to better solve the inter-turn oscillation $[10,11]$. In this paper, a simulation circuit for high-frequency impulse effect was established based on the multi-conductor transmission line (MTL) theory. The circuit could simulate and verify the transient voltage distribution along winding, voltage between turns and voltage of nodes to earth. In addition, the simulation results can help to assess the damage of transformer insulation and adjust transformer insulation design flexibly.

\section{Model of Transformer Windings}

2.1 MTL Theory. For continuous-type transformers, the windings were turned on at each turn, and the entire winding became a multi-coupled and end-to-end transmission line, so that the winding could be modeled as a loss uniform multi-conductor transmission line [12]. Figure 1 displays the model of the transformer windings by its individual turns, where $U_{S}(i), I_{S}(i), U_{L}(i)$ and $I_{L}(i)$ represents the voltage and current vectors at the beginning and end of the conductor correspondingly. The model could also be described by the following equation:

$$
\begin{aligned}
& \frac{d \mathbf{U}}{d x}=-(\mathbf{R}+j \omega \mathbf{L}) \mathbf{I} . \\
& \frac{d \mathbf{I}}{d x}=-(\mathbf{G}+j \omega \mathbf{C}) \mathbf{U} .
\end{aligned}
$$

where $\mathbf{U}$ and $\mathbf{I}$ are the voltage and current vectors at position $\mathrm{x}$ along the lines; $\mathbf{R}, \mathbf{L}, \mathbf{G}$, and $\mathbf{C}$ are the per unit length resistance, inductance, conductance, and capacitance matrix, respectively. 
According to [12], the transfer function from the terminal of arbitrary turn $\mathrm{k}$ to the input is

$$
\begin{aligned}
A(k) & =\frac{U_{R}(k)}{U_{S}(1)} \\
& =\frac{T(k+1,1) T(N+1, N+1)-T(k+1, N+1) T(N+1,1)}{T(1,1) T(N+1, N+1)-T(N+1,1) T(1, N+1)} .
\end{aligned}
$$

where $\hat{Y}$ is a $(\mathrm{N}+1) \times(\mathrm{N}+1)$ matrix, and $T=\hat{Y^{-1}}$.

According the equation 2, we can calculate the voltage distribution of the transformer windings at any time.

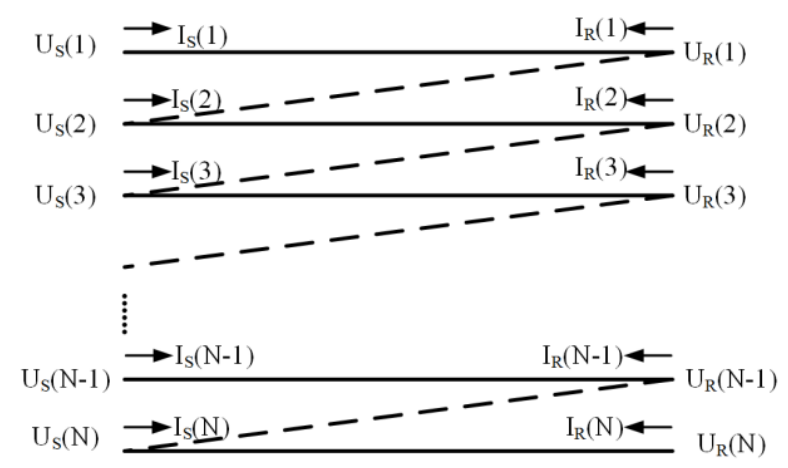

Fig. 1. Multi-conductor transmission line model

2.2 The Equivalent Circuit Model. As turns should be considered and this leads to a large number of equations and solving the problem requires very large matrix operations, the equivalent circuit built up in turn is too complex to simulate and calculate. When the coil length of each line disk is far less than $1 \%$ of the wavelength of lighting, an equivalent circuit of transformer winding based on the disk unit was established, as shown in Figure 2.

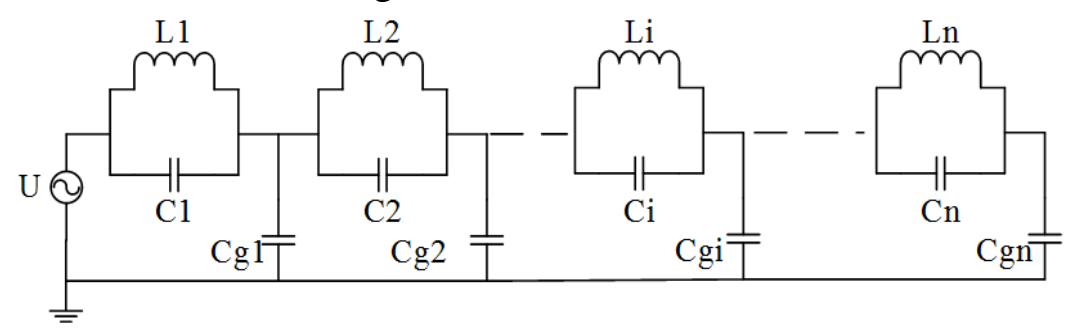

Fig. 2 Circuit diagram for simulation

The equivalent circuit is constituted of continuous lumped parameter components such as L1, C1, $\mathrm{Cg} 1$ which are usually used to study the transient response of windings. In Fig2, L1, L2,.., Ln represent inductance along the windings, and $\mathrm{Cg} 1, \mathrm{Cg} 2, \ldots, \mathrm{Cgn}, \mathrm{C} 1, \mathrm{C} 2, \ldots, \mathrm{Cn}$ respectively represent the capacitance to ground along the windings and inter-turn capacitance. Therefore, taking the disk as unit the model of transform wind-ings was established.

In this paper, the transient voltage was defined for standard lightning impulse $(1.2 / 50 \mu \mathrm{s})$, and the amplitude was $2 \mathrm{MV}$. The simulation transformer has 108 disks, and the part of the equivalent parameters is shown in Table 1. The capacitance of Cgi in the model is $40 \mathrm{pF}$.

\begin{tabular}{|c|c|c|c|c|c|c|c|c|}
\hline & $\mathrm{H}$ & $\mathrm{H}$ & $\mathrm{L}_{4}$ & $\mathrm{H}$ & $\mathrm{L}_{6}$ & $\mathrm{~L}_{7}$ & & \\
\hline & & & & & & & & \\
\hline $\mathrm{C}_{1} /$ & & & & & $\mathrm{C}_{6} / \mathrm{pF}$ & & & $\mathrm{C}_{9} / \mathrm{pF}$ \\
\hline 8265 & 8265 & 3397 & 1454 & $2-$ & 30 & 300 & 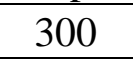 & 300 \\
\hline
\end{tabular}

Table 1. Equivalent parameter of transformer winding 


\section{Results and Discussion}

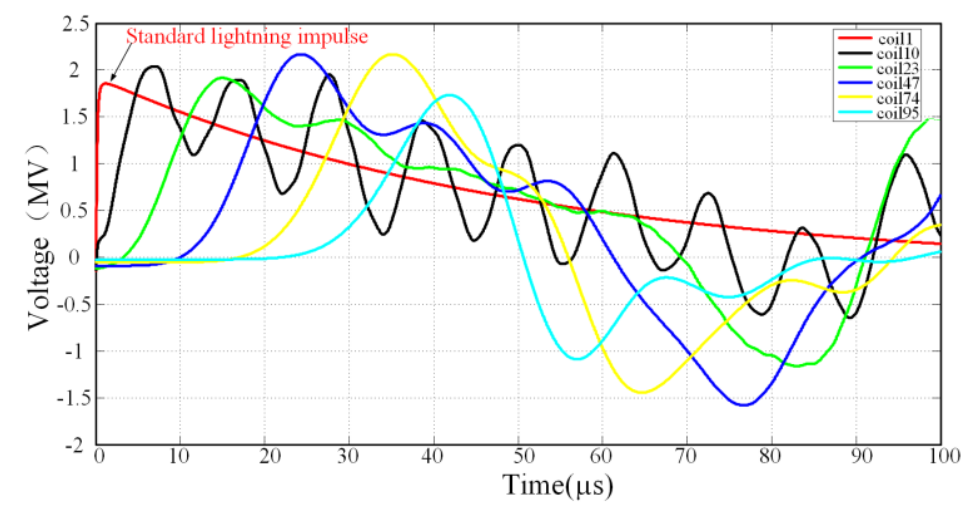

Fig. 3 Transient Voltage Waveform under Standard Lightning

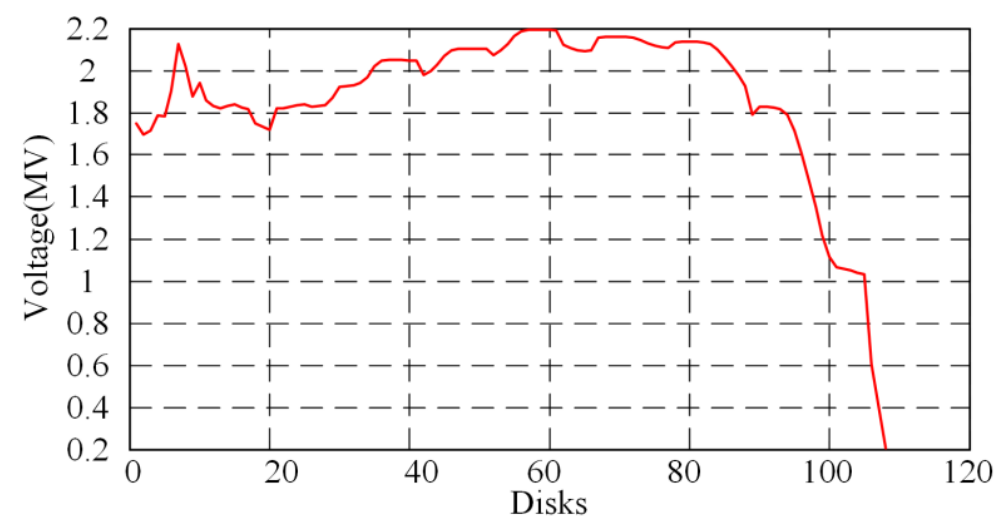

Fig. 4 Voltage distribution of each disk

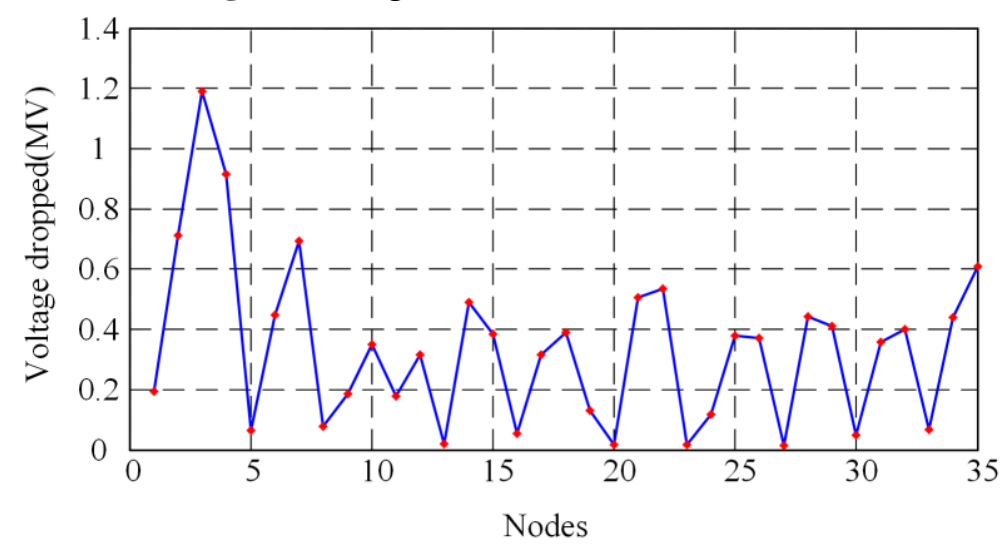

Fig. 5 Voltage drop between disks

The voltage distributions of several nodes are shown in Figure 3. The simulation results manifested that the voltage at the No. 74 is the largest and the voltage amplitude of the middle part is higher than that of the first part.

The peak voltage distribution of each disk under the lightning wave was shown in Fig 4. And Fig 5 presented the voltage drop between the disks. The simulation results indicated that the maximum voltage was in the middle of the winding under the lightning impulse, and the maximum voltage drop between disks appeared the beginning of the winding.

\section{Summary}

ATP-EMTP simulation are used to calculate the distribution of transient voltage in electric power transformer windings. When the lightning impulse imposed at the first turn of coils, the maximum voltage is in the middle layer winding disk, and the maximum voltage gradient is appeared in the first section of the winding. The simulation results demonstrated the transformer equivalent circuit used in 
this paper is suitable to analyze the voltage distribution on the transformer windings under transient impulse.

\section{References}

[1] Shibuya, Y., S. Fujita, and T. Shimomura, in: Effects of very fast transient overvoltages on transformer, edit by IEE Proceedings-Generation, Transmission and Distribution 146.5 (1999): 459-464.

[2] Shibuya, Yoshio, Shinobu Fujita, and N. Hosokawa, in: Analysis of very fast transient overvoltage in transformer winding, edit by IEE Proceedings-Generation, Transmission and Distribution 144.5 (1997): 461-468.

[3] Popov, Marjan, in: Computation of very fast transient overvoltages in transformer windings, edit by IEEE Transactions on Power Delivery 18.4 (2003): 1268-1274.

[4] Ying, Peng, and Ruan Jiangjun, in: Investigation of very fast transient overvoltage distribution in taper winding of tesla transformer, edit by IEEE Transactions on Magnetics 42.3 (2006): 434-441.

[5] Petrarca, Carlo, in: Analysis of the voltage distribution in a motor stator winding subjected to steep-fronted surge voltages by means of a multiconductor lossy transmission line model, edit by IEEE transactions on Energy Conversion 19.1 (2004): 7-17.

[6] Woivre, V., in: Transient overvoltage study and model for shell-type power transformers, edit by IEEE Transactions on Power Delivery 8.1 (1993): 212-222.

[7] Moreau, O. , in: 3-D high frequency computation of transformer R, L parameters, edit by IEEE Transactions on Magnetics 41.5 (2005): 1364-1367.

[8] Malewski, Ryszard, Michael A. Franchek, and James H. McWhirter, in: Experimental validation of a computer model simulating an impulse voltage distribution in HV transformer windings, edit by IEEE transactions on power delivery 9.4 (1994): 1789-1798.

[9] Gharehpetian, G. B., H. O. S. S. E. I. N. Mohseni, and K. Moller, in: Hybrid modelling of inhomogeneous transformer winding for very fast transient overvoltage studies, edit by IEEE Transactions on Power Delivery 13.1 (1998): 157-163.

[10] Wang, Z, in: A model for transient analysis in large transformer windings, edit by Journal of Tsinghua University 33.1 (1993): 25-32.

[11] Yang, Y., and Z. J. Wang, in: A circular multi-conductor transmission line model for simulation of very fast transient in circular windings, edit by Proceedings of the Progress In Electromagnetics Research Symposium. 2009.

[12]Liang, Guishu, in: Modeling of transformer windings under very fast transient overvoltages, edit by IEEE Transactions on Electromagnetic Compatibility 48.4 (2006): 621-627. 